\title{
Jean-Claude Dreyfus (1916-1995)
}

\author{
Axel Kahn
}

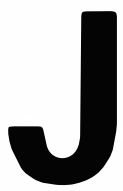

ean-Claude Dreyfus était des nôtres lorsque nous avons fêté les dix ans de médeci$n e /$ sciences, lui qui était l'un des membres fondateurs de notre Comité de Rédaction, infatigable rédacteur et chroniqueur de talent. Aujourd'hui, il n'est plus. Ses écrits sont pourtant encore nombreux dans ce numéro et le seront dans notre numéro du mois de juillet. C'est que la présentation à nos lecteurs de l'actualité scientifique en génétique, la rédaction de nouvelles et de brèves, fut un travail qu'il mena sans relâche jusqu'à quelques heures de son décès subit, au matin du 10 mai.

Écrire pour et se préoccuper de médecine/sciences sont d'ailleurs parmi les toutes dernières actions et pensées de Jean-Claude Dreyfus qui était prodigieusement attaché à notre revıe, et médecine/sciences lui devait beaucoup.

Quelle merveille de voir cet homme de 79 ans se mouvoir avec une stupéfiante agilité intellectuelle dans les arcanes de la biologie moléculaire moderne, ses techniques complexes et évolutives, sa progression étourdis-

$\mathrm{m} / \mathrm{s} n^{\circ} 6$, vol. 11 , juin 95

C'est après son départ à la retraite de l'unité INSERM qu'il dirigeait depuis 10 ans que Jean-Claude Dreyfus put se consacrer presque entièrement à notre revue. Auparavant, il fut un scientifique de tout premier plan, co-fondateur avec Georges Schapira, son presque frère jumeau sur le plan de l'histoire et de la carrière, de la Pathologie Moléculaire en France. Reçu premier au § concours de l'Externat des Hôpitaux de Paris en 1936, Jean-Claude Dreyfus était rapidement interne, nommé à son premier concours en 1938, juste avant un service militaire qui se termi-

sante ! Il rendait compte de la fantastique aventure scientifique de la génétique moderne avec une clarté et un esprit de synthèse en disant long sur ses capacités cognitives au service desquelles il mettait aussi une mémoire stupéfiante. Il y investissait un enthousiasme et une capacité d'émerveillement témoignant d'une juvénilité mentale conservée. Conservée, elle l'était d'ailleurs jusque dans les détails de son comportement, de cette timidité entrecoupée de remarques acérées et pénétrantes, parfois d'un humour dévastateur. 
dans une rafle à Annecy, où il fêtait son anniversaire, et déporté en décembre 1943, heureusement sous son identité d'emprunt. Ce furent alors les camps de concentration de Compiègne, Buchenwald, Dora et ses tunnels où se fabriquaient les armes de la dernière illusion du Reich, les fusées V2. C'est le $1^{\text {"r }}$ mai 1945 que Jean-Claude Dreyfus revint à Paris; il neigeait.

Ce fut alors le début de temps moins troublés où l'homme forgé dans l'épreuve, marqué par elle, put s'épanouir dans la plénitude de sa créativité scientifique.

Dès 1945, Georges Schapira et JeanClaude Dreyfus fondaient avec Fanny Schapira, d'abord dans une salle d'opérations désaffectée, puis dans les sous-sols de la clinique médicale infantile du Professeur Robert Debré, le premier laboratoire de médecine moléculaire en France, l'un des premiers au monde. Sous la direction de Georges Schapira, le petit groupe devait s'enrichir progressivement de personnalités marquantes, artisans eux aussi du développement de la Pathologie Moléculaire dans notre pays: Jacques Kruh, à qui, par une ironie cruelle de la vie et de la mort, Jean-Claude Dreyf us rendait hommage dans notre numéro d'avril, Prudent Padieu, Dominique Labie, Raymond Saddi, Jean Démos, Jean Rosa, Raymonde Rosa, Jean-Claude Kaplan, Jacques Hanoune, Yves Nordmann.... et la liste ne saurait être complète. En 1967, l'Institut de Pathologie Moléculaire était fondé grâce au soutien de Robert Debré et de Jacques Monod; il s'installera en 1968 dans les nouveaux locaux universitaires de la Faculté de Médecine Cochin PortRoyal.

Le déménagement des laboratoires exigus du sous-sol des EnfantsMalades dans le nouvel Institut, Unité 15 de l'INSERM et laboratoire associé au CNRS, devait permettre d'accueillir de nouveaux chercheurs, de nouvelles équipes parmi lesquels on peut citer les noms de Claudine Junien, Livia Poenaru, Bernard Dastugue, Jean Delaunay, Jacques Elion, Marc Delpech... et moi-même. Le développement de l'Institut justifiait,

I'INSERM. Jean-Claude Dreyfus prit alors la direction du laboratoire d'enzymologie pathologique, Unité 129 de l'INSERM, jusqu'à sa retraite en 1984, date à laquelle je pris sa succession. Jean-Claude Kaplan, quant à lui, lui succédait sur les plans hospitalier et universitaire.

Les premiers travaux de Jean-Claude Dreyfus, menés en collaboration avec Georges et Fanny Schapira, portent sur le métabolisme du fer et les maladies musculaires, myopathies et atrophies neurogènes, avec deux découvertes de grande importance: l'élévation de l'activité d'enzymes glycolytiques musculaires dans le sérum des myopathes et de nombre de femmes porteuses, et la résurgence d'isoenzymes foetales dans le muscle myopathe. Des travaux sur l'hémochromatose précisent sa transmission héréditaire et permettent de développer de nouveaux tests diagnostiques. La biosynthèse de l'hémoglobine est étudiéc avec Jacques Kruh, et d'importantes observations sont faites sur les maladies moléculaires de l'hémoglobine, avec défaut qualitatif ou quantitatif. Ce sujet sera ensuite poursuivi par Dominique Labie et Jean Rosa. Avec Fanny Schapira et Yves Nordmann sont découvertes les résurgences d'isoenzymes foetales dans le cancer, progrès considérable qui attire pour la première fois l'attention sur les relations entre le programme génétique de différenciation et la cancérisation. Bien plus tard, ce type d'analyse sera appliqué à l'étude du vieillissement; cette recherche aboutira à l'identification de deux phénomènes associés au vieillissement cellulaire: une altération post-traductionnelle des protéines, liée au ralentissement de leur renouvellement, et quelques modifications de l'expression du programme de différenciation.

Cependant, l'essentiel de l'œuvre scientifique de Jean-Claude Dreyfus a été consacré aux maladies enzymatiques héréditaires, cette orientation étant une suite logique du séjour d'un an accompli aux États-Unis, en 1953, dans le laboratoire des Cori, qui découvrirent en 1938 l'interconversion des formes active et inactive de la phosphorylase musculaire et obtinrent le prix Nobel pour cette découverte en 1947. Carl et Gerti Cori avaient aussi laissé leur nom à une classification des glycogénoses, toujours en vigueur.

L'activité de Jean-Claude Dreyfus dans ce domaine de l'enzymologie pathologique recouvrit donc les glycogénoses, mais aussi les maladies lysosomiales en général, les déficits enzymatiques érythrocytaires et, avec Fanny Schapira, les affections du métabolisme du fructose et du galactose. Avec Georges et Fanny Schapira, il a introduit en France une grande partie des techniques enzymatiques utilisées en biochimie clinique et a créé, en 1973, le premier laboratoire français de détection prénatale des maladies métaboliques, réalisant lui-même une partie des dosages durant de longues heures pleines d'angoisse au terme desquelles il lui était souvent difficile de trouver le sommeil. Il faut ici citer l'aide apportée à l'entreprise par Joëlle et André Boué et par Yves Dumez, pionnier des techniques de prélèvement de tissu fœeto-placentaire en France.

Sur le plan théorique, Jean-Claude Dreyfus a analysé dès 1968, et avec une étonnante lucidité, les conditions de l'application aux maladies génétiques humaines des concepts de la génétique bactérienne définis en 1961 par François Jacob et Jacques Monod. Alors qu'une division arbitraire des mécanismes des maladies génétiques en "anomalies de gènes de structure " et "anomalies de gènes de régulation " faisait autorité depuis 1963 et était reprise dans la «bible» qu'est le "Stanbury-Wyngaarden-Frederickson " (édition 1966), Jean-Claude Dreyfus observait dans un article fameux publié en 1969 (The application of bacterial genetics to the study of human genetic abnormalities in Steinberg A \& Bearn AG, eds. Progress in medical genetics, New York, Grune and Stratton 1969, vol. 6, p 169-200) que la plupart des affections dans lesquelles le produit d'un gène n'est pas détecté ou est en quantité diminuée sont génétiquement "codominantes", ce qui signifie qu'un hétérozygote à des anomalies intermédiaires entre celles d'un homozygote normal et d'un homozygote mutant. Par conséquent, l'ano- 

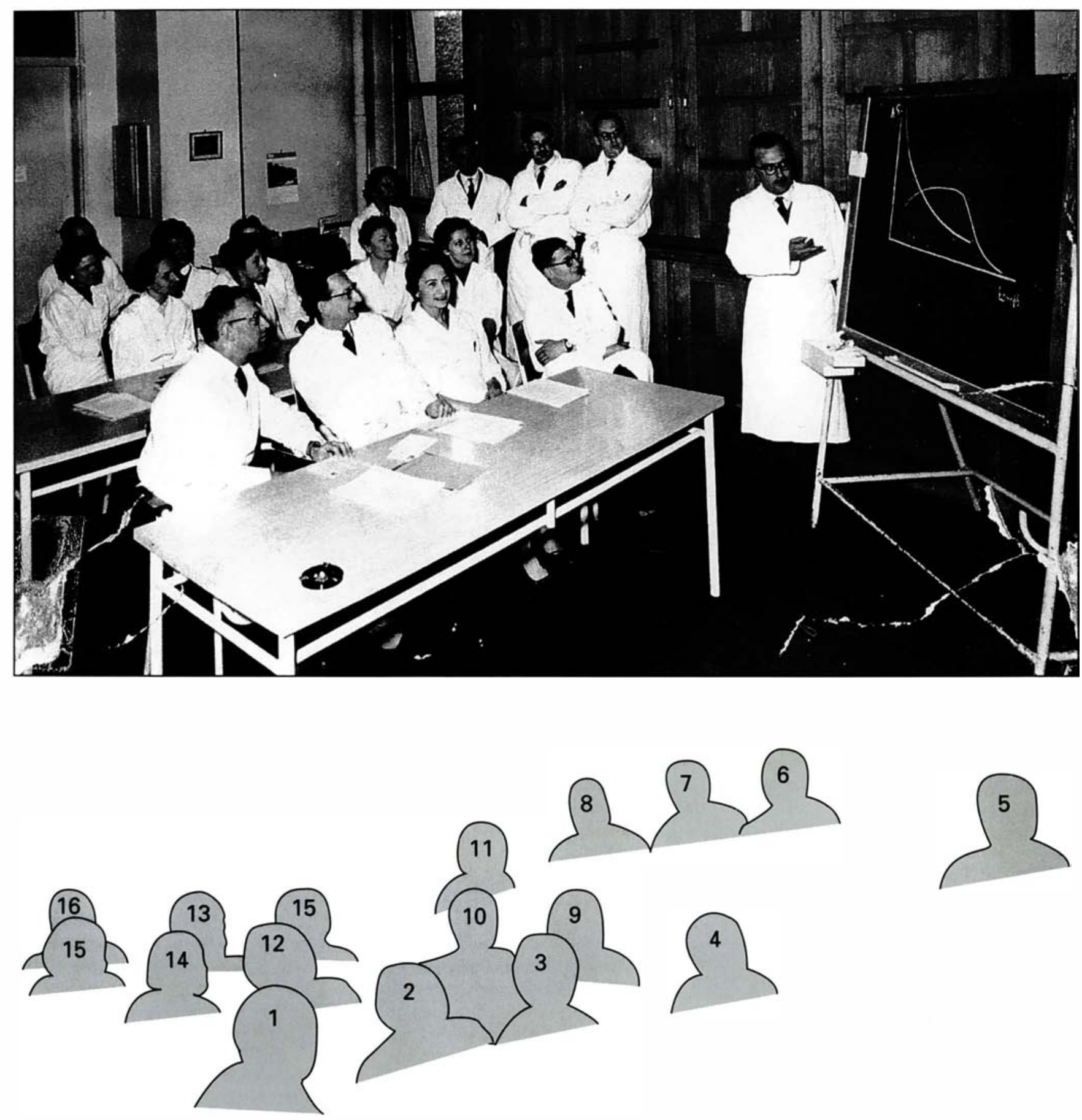

1. Jean-Claude DREYFUS - 2. Georges SCHAPIRA - 3. Fanny SCHAPIRA - 4. Jean ROSA - 5. Jacques KRUH 6. Jean de GROUCHY - 7. Prudent PADIEU - 8. Jean DEMOS - 9. Gilberte DEMOS - 10. Paulette AFFICHARD 11. Marie-Louise CROLAND - 12. Lise MARCAUD-RAEBER - 13. Magdeleine VIBERT - 14. Lydie TICHONICKY 15. Yvonne ALEXANDRE - 16. Denis DAGUILLON

En 1958, dans le laboratoire du sous-sol de la Clinique Médicale Infantile de l'hôpital des Enfants Malades. 
malie génétique doit se situer en cis du gène concerné, ce qui exclut des mutations régulatrices agissant en trans, par exemple la synthèse d'un super-répresseur (dominance vraie, les hétérozygotes ayant les mêmes anomalies que les homozygotes mutants) ou la carence en un activateur (récessivité vraie, les hétérozygotes n'ayant aucune anomalie biochimique détectable). Restent donc, pour expliquer les maladies génétiques humaines, les mytations de la séquence codante (structurales) ou les mutations de type "opératrices" pour reprendre la terminologie de la génétique bactérienne. De telles prédictions, précédant de plus de cinq ans l'avènement du génie génétique, sont remarquables et devaient pour l'essentiel être confirmées par l'identification des mutations en cause dans les maladies génétiques, au détail près que des mutations d'épissage, qui ne pouvaient d'aucune manière être imaginées à l'époque, sont plus souvent en cause dans les anomalies quantitatives d'expression des gènes que les mutations des séquences régulatrices actives en cis. Les vraies mutations de régulation actives en trans sont exceptionnelles chez l'homme; on peut y inclure certains défauts d'expression des molécules de classe II du complexe majeur d'histocompatibilité dans des déficits immunitaires congénitaux $\left(\mathrm{m} / \mathrm{s} n^{\circ} 4\right.$, vol. 5, p. 268) et le syndrome de retard mental associé à une thalassémie $\alpha$ liée au chromosome X $\left(m / s n^{\circ} 6\right.$, vol. 11, p. 910). Toutes ces idées étaient reprises, et développées, plus de 20 ans après, dans un article publié dans médecine/sciences (Mécanismes moléculaires des enzymopathies génétiques. médecine/sciences 1989; 5:93-102).

Jean-Claude Dreyfus était aussi Professeur de Biochimie Médicale d'abord à Dijon, puis à la Faculté de Médecine de Cochin. Il fut membre d'une Commission Scientifique
Spécialisée (1964-1968) et du Conseil Scientifique (1969-1974) de l'INSERM, du Comité de Biologie Moléculaire de la DGRST (19631967) et du Conseil Scientifique de l'Association Contre les Myopathies (AFM) dans les années 1980. Il était président d'honneur de l'association VML (Vaincre les Maladies Lysosomiales).

Un élément d'étonnement est qu'un tel scientifique, auteur d'une telle œuvre, n'ait pas bénéficié d'une notoriété et d'un rayonnement encore plus importants et universels que ceux qu'il connut.

Ce qu'il en a fallu de réserve, parfois de timidité de sa part, pour masquer à la plupart ce qui ne fait aucun doute pour ses proches, à savoir que Claude, comme l'appelaient ses intimes, était l'un des hommes les plus prodigieusement intelligents qui soient, l'un des scientifiques les plus visionnaires qu'il m'ait été donné d'approcher.

Cet homme de culture était aussi un grand humaniste conformant sa vie à son éthique et prêt à s'engager en des combats généreux, toujours associé alors à Colette Dreyfus-Brisac, son épouse depuis 1948. On les vit tous deux lutter contre les guerres coloniales et impérialistes et s'efforcer de venir en aide aux réfugiés, notamment des professions médicales et paramédicales, d'où qu'ils viennent: Chili et autres pays d'Amérique Latine, Vietnam et autres pays du Sud-Est asiatique, pays d'Europe, Afrique noire, etc. Jean-Claude Dreyfus aimait les gens, et conserva un contact paternel avec ses élèves bien au-delà de sa retraite officielle, s'intéressant à leur recherche, à leur carrière, à leur vie. Il avait deux filles, Catherine et Martine, et quatre petits-cnfants dont il me parlait souvent, avec l'évidence d'un amour paternel et grand patemel dont il ne rougissait pas de faire partager l'intensité à ses amis. Jean-Claude Dreyfus était de ces per- sonnalités dont l'éclat illumine la destinée de ses proches, de ses élèves et continuateurs, et laisse ainsi une trace permanente. J'ai été son interne, en 1972, dernière année de mon internat que j’avais réservée chez Georges Schapira quelques minutes après que $\mathrm{j}$ 'ai appris mon succès au concours. Je me rappelle son plaisir manifeste, à lui qui était l'antithèse du mandarin, lorsqu'il me présentait à des collègues comme "son interne...". Très rapidement, cependant, nos relations furent celles d'élève à maître, et on ne dit plus assez la grandeur et la beauté de cette relation là, qui est attente, souvent exigeante, confiance, considération et, de la part de l'élève, aspiration à s'élever au niveau du maître. Ce maître, je l'aimais et l'admirais même un peu plus qu'il n'est coutume de la part d'un élève, et je crois bien qu'il $y$ avait quelque chose de filial dans mon attachement, peut-être quelque chose de paternel dans son sentiment.

La succession provoquée par son départ à la retraite en 1984 ne fut pas un traumatisme tant Claude restait proche de l'évolution de son unité, tant nous continuions de travailler de concert... notamment à médecine/ sciences.

Jean-Claude Dreyfus a profondément marqué la formation, parfois la vie, de ses proches, les infléchissant en un sens qu'il ne viendrait à l'esprit de personne de récuser. En leur nom comme au mien, en forme de dernier hommage, je voudrais dire merci à Jean-Claude Dreyfus ... merci d'avoir existé et d'avoir été ce qu'il fut

\section{Axel Kahn}

Directeur de l'Inserm U. 129. IGGM, unité de génétique et de pathologie moléculaires, Inserm U.129, 24, rue du Faubourg-Saint-Jacques, 75014 Paris, France. 